\title{
Internet Remotely-Operated Experiments for Educational Purposes
}

Mona Mobasher, Ashraf Kamal, Mohamed Mahsoub

\begin{abstract}
The purpose of this paper is to present Blended learning in physics laboratory and use of technology which includes enhancements to teaching materials, student questionnaires, computer aided examples and virtual experiments. Three main approaches are covered in this paper Hands-on Labs, Virtual Labs and Remote Labs. Hands-on experiments enhance learning at different levels of engineering education as they allow students to operate a real experiment, while Virtual Labs are defined as a computer-based model of the real labs, and Remote Labs are achieved using the internet. Another aspect developed in this paper which discusses the technical considerations of hardware-based experiments and their implementation in an internet-based remote laboratory environment. Undergraduate students' experiments are introduced as examples implemented in the remote laboratory. Statistics of students' grades are obtained for evaluating the progress of laboratories. A detailed look inside the platform of remote protocol is introduced in this paper
\end{abstract}

